\title{
Characteristics of Dielectric Barrier Discharge Reactor for Material Treatment
}

\author{
K.G. Kostov, R. Y. Honda, L.M.S. Alves, and M.E. Kayama \\ Engineering Faculty of Guaratinguetá FEG, State University of São Paulo UNESP, \\ Av. Dr. Ar. P. da Cunha 333, 12516-410 Guaratinguetá, SP, Brazil
}

(Received on 6 March, 2009)

\begin{abstract}
This work reports the development of atmospheric pressure plasma reactor with dielectric barrier discharge DBD for material treatment. The DBD discharge has been generated in planar geometry reactor powered by ac voltage provided by conventional high voltage transformer. The dielectric barrier consisted of two glass slabs, which cover both reactor electrodes. The air discharge gap between the dielectric layers was varied from 1.0 to $3.0 \mathrm{~mm}$. The power consumption of the DBD reactor was evaluated by the Lissajous figures method. The optimization of reactor geometry for material processing is discussed.
\end{abstract}

Keywords: DBD discharge, Plasma reactor, Electric circuit model

\section{INTRODUCTION}

There are two widely used methods for generating nonthermal plasmas at atmospheric pressure, namely the corona discharge and the dielectric barrier discharge DBD. Corona discharges have many applications in industry, where relatively small concentrations of excited or charged species are needed. Typical examples are electrostatic precipitators, copying machines, dry-ore separation systems and highspeed printout devices, where corona discharges are used to produce charged particles. On the other hand, the DBD discharges are predestined for applications of large volume plasma chemistry. Barrier discharges, also referred as silent discharges are characterized by the presence of at least one insulating layer between two planar or cylindrical electrodes connected to an ac power supply [1]. The main advantage of this type of electrical discharge is that non-equilibrium plasma conditions in atmospheric pressure gases can be established in an economic and reliable way. This feature has led to a number of important DBD applications including industrial ozone generation [1], pollution control [2], plasmachemical vapour deposition and surface activation [3], excitation of excimer lamps, and more recently surface modification of diverse materials [4].

In order to optimise the design of a DBD rector, there is a necessity to calculate the power consumed in the silent discharge over a wide range of discharge conditions. In some previous studies [5-8], the authors have investigated the silent discharge current-voltage characteristics in coaxial wire-cylinder reactor aimed for ozone generation. On the other hand, reactors with parallel plate geometry are more suitable for material processing. Therefore, in this work the discharge power consumed in a parallel plate DBD reactor with two dielectric layers has been measured as a function of the applied voltage and the discharge gap. A simplified model of the reactor equivalent electrical circuit is used to calculate the consumed power and it is shown that there exists an optimal discharge gap. For this distance the electric power delivered to the discharge is maximal, which is advantageous for material processing applications. Reactor power measurements demonstrated that the maximal discharge power is achieved at discharge gap of $1.5 \mathrm{~mm}$, which is in a good agreement with the prediction from the equivalent circuit model.

\section{ANALYSIS OF DBD REACTOR ELECTRICAL CIRCUIT}

The equivalent electric circuit of parallel plate DBD reactor with two dielectric layers over the metal electrodes is shown in Fig. 1.
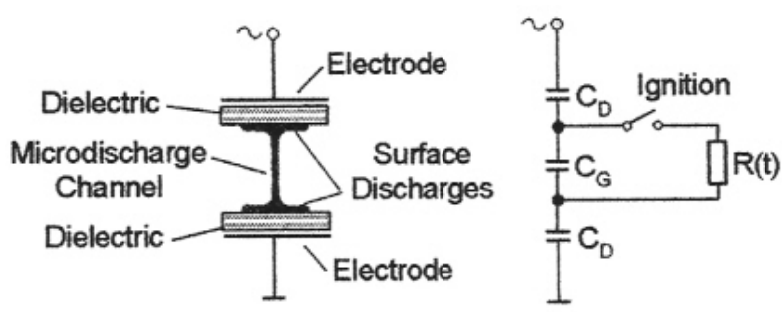

FIG. 1: Layout of DBD reactor equivalent circuit.

The complex impedance $Z$ of the circuit shown in Fig. 1 is given by:

$$
Z=\frac{R}{\left(1+\omega^{2} C_{G}^{2} R^{2}\right)}-j\left(\frac{2}{\omega C_{D}}+\frac{\omega C_{G} R^{2}}{1+\omega^{2} C_{G}^{2} R^{2}}\right)
$$

where $C_{D}=\frac{\kappa \varepsilon_{0} A}{d}$ is the capacitance of the dielectric layers; $C_{G}=\frac{\varepsilon_{0} A}{x}$ is the capacitance of the air gap and $R$ is the equivalent resistance of the DBD discharge. Here $A$ is the electrodes area, $d$ is the thickness of the dielectric layer, $k$ is the dielectric constant and $x$ is the discharge gap width.

Then the mean electric power consumed for one oscillation period is given by:

$$
\bar{P}=\frac{1}{2} \frac{V^{2}}{|Z|} \cos \varphi=\frac{1}{2} \frac{V^{2}}{|Z|} \frac{\operatorname{Re}(Z)}{|Z|}
$$

where $V$ is the ac voltage amplitude and $\varphi$ is the phase angle between the tension and the current.

Substituting the real part of the impedance $Z$ and its module into (2) one gets

$$
\bar{P}=\frac{V^{2}}{2 R}\left[\left(1+4 \frac{C_{G}}{C_{D}}+4 \frac{C_{G}^{2}}{C_{D}^{2}}\right)+\frac{4}{\omega^{2} C_{D}^{2} R^{2}}\right]^{-1}
$$


Assuming that DBD discharge is constituted by various micro-discharges in parallel the plasma resistance can be approximately expressed as $R \cong x \rho / A_{E F}$, where $\rho$ is the resistivity of a discharge channel and $A_{E F}$ is the total cross section occupied by the micro-discharges. The mean reactor power can be expressed in term of the discharge gap $x$.

$$
\bar{P}=\frac{V^{2}}{2}\left\{\frac{\rho}{A_{E F}}\left[\frac{4 d}{\kappa}+x+\frac{4 d^{2}}{\kappa^{2}}\left(1+\frac{A_{E F}^{2}}{\varepsilon_{0}^{2} \rho^{2} \omega^{2} A^{2}}\right) \frac{1}{x}\right]\right\}^{-1}
$$

From the condition for maximum of the discharge power $\frac{d \bar{P}}{d x} \equiv 0$ using $x$ as a variable one can obtain the value of the discharge gap $x_{\text {max }}$, which maximizes the reactor power:

$$
x_{M A X}=\frac{2 d}{\kappa} \sqrt{1+\frac{A_{E F}^{2}}{\rho^{2} \omega^{2} \varepsilon_{0}^{2} A^{2}}}
$$

Generally at fixed AC voltage the discharge current scales with the electric field $E$ in the gap $(I \propto E \propto 1 / x)$, so it is expected an increase of the discharge power as the reactor air gap is decreasing. However, the reactor total capacitance and correspondingly the phase difference $\varphi$ between the AC voltage and the discharge current are also inversely proportional to the distance $x$. Since the AC power delivered to the silent discharge depends on the phase factor $\cos \varphi$ at very short discharge gaps (smaller than $x_{M A X}$ ) the reactor power is expected to decrease.

\section{EXPERIMENTAL}

The experimental arrangement used to study DBD discharge at atmospheric pressure is sketched in Fig. 2. The discharge is generated between two plane electrodes, 4.0 $\mathrm{cm}$ in diameter which are covered by $5.0-\mathrm{mm}$-thick glass layers. The high voltage power supply is consisted of a step-up transformer $(100 / 20000 \mathrm{~V}$ of Vrms, $60 \mathrm{~Hz})$, an autotransformer Variac and a load resistor of $1 \mathrm{k} \Omega, 500 \mathrm{~W}$. The $A C$ voltage applied to the reactor upper plate is measured by using a 1000:1 high-voltage probe (Tektronix P6015A, $75 \mathrm{MHz}$ ) and displayed on a two-channel digital oscilloscope (Tektronix TDS 320, 100MHz).

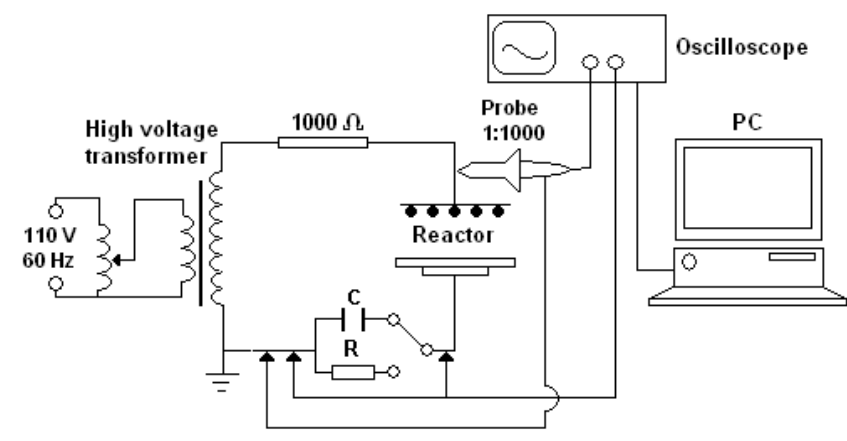

FIG. 2: Experimental setup.

For displaying the waveform of the discharge current on the oscilloscope the reactor down plate was grounded through a current measurement resistor of $1200 \Omega$. A simple and reliable method for obtaining the consumed power is using the discharge Lissajous figures, obtained when plotting transported electric charge $\mathrm{Q}$ through the discharge as a function of the applied periodical voltage $[9,10]$. Experimentally, the charge $Q$ is delivered from the voltage drop across a measuring serial capacitor of $0.91 \mu \mathrm{F}$. The average electric energy $W$ dissipated in a discharge cycle is then simply the area of the characteristic Lissajous figure, which in most cases is nearly a parallelogram [11]. Then the mean discharge power is calculated according to the relation $P=f$ $W$.

\section{RESULTS AND DISCUSSION}

Figure 3 shows waveforms of the voltage applied to the reactor and the associated discharge current at $2.0 \mathrm{~mm}$ reactor gap. When the AC voltage applied to the DBD reactor reaches the onset value, the discharge starts in the air gap inside the reactor in the form of filamentary streamers. The filaments are randomly distributed over entire electrode surface. The streamers cross the discharge gap and spread on the surface of the dielectric barrier, building up surface charges, which produce electric field opposite to that of the applied voltage. So after short time (several ns) the streamer activity in that spot is extinguished, followed by streamer initiation in another location. At the peaks of the applied ac voltage, where $d V / d t=0$, the displacement current across the dielectric is zero and streamer activities cease to exist. Streamers start again when the voltage applied to discharge gap reaches the onset value during the next half-cycle.

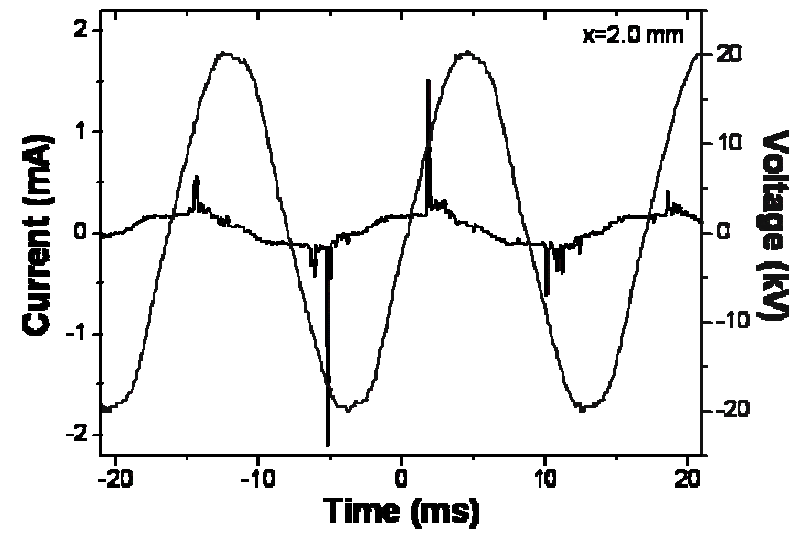

FIG. 3: Waveform of the discharge current of DBD reactor with discharge gap width of $2.0 \mathrm{~mm}$.

The effect of the reactor geometry on the discharge power is analysed by the Lissajuos figures. Figures $4 a-b$ show the Q-V characteristics of the DBD reactor for different discharge gap $x$. Fig. 4(a) depicts the Lissajous figures taken at gap widths of 1.0 and $2.0 \mathrm{~mm}$. Both figures have approximately the form of parallelogram with about the same area but different inclination.

The sides $\mathrm{AB}$ and $\mathrm{CD}$ of the Lissajous figure represent 


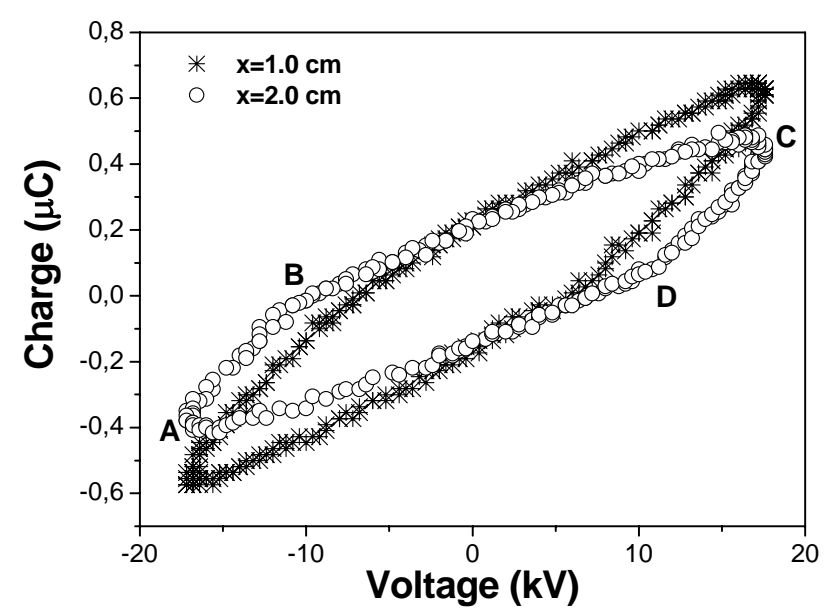

Fig.4a. Influence of the reactor gap width on the shape of Lissajous figures.

the burning discharge, where the air gap is partially shortcut. These sides of the both figures are almost parallel but the one corresponding to $1.0 \mathrm{~mm}$ gap is longer since the discharge onset at shorter distance occurs at lower voltage. On lines BC and DA, where there is only displacement current, the slope $d Q / d V$ corresponds to the total reactor capacitance. As predicted by the model the reactor capacitance decreases with the gap distance, leading to change of Lissajous figures shape.

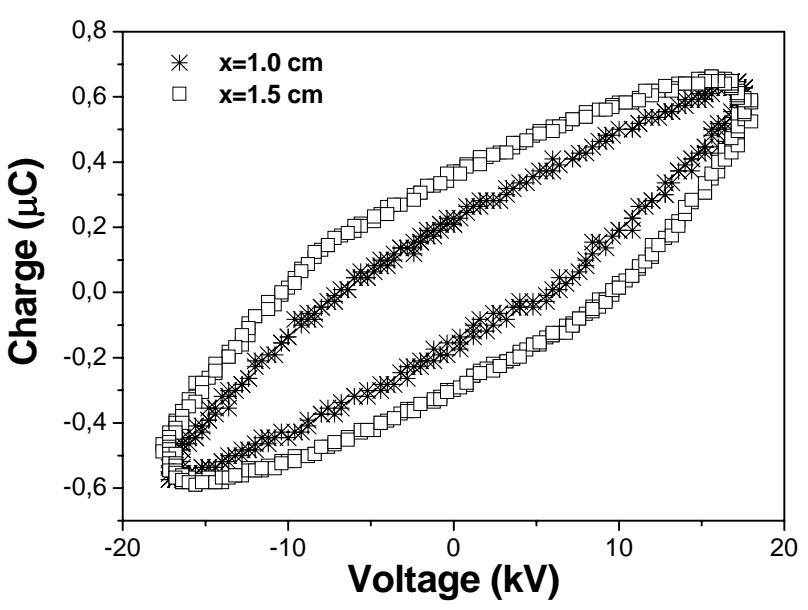

Fig.4b. Influence of the reactor gap width on the area of Lissajous figures.

As shown in Fig. 4(b) however, the area of the Q-V figure significantly increases when the gap width is changed from 1.0 to $1.5 \mathrm{~mm}$. This finding is in a good agreement with the simplified electric circuit model of the discharge power (4), which predicts that there should be a power maximum at certain gap distance $x_{M A X}$.

The average discharge power, calculated from the Lissajous figures, versus the applied ac voltage is plotted in Fig. 5 for the gap widths used in Fig. 4. As can be seen from this figure the power consumption for the gap distance of 1.5

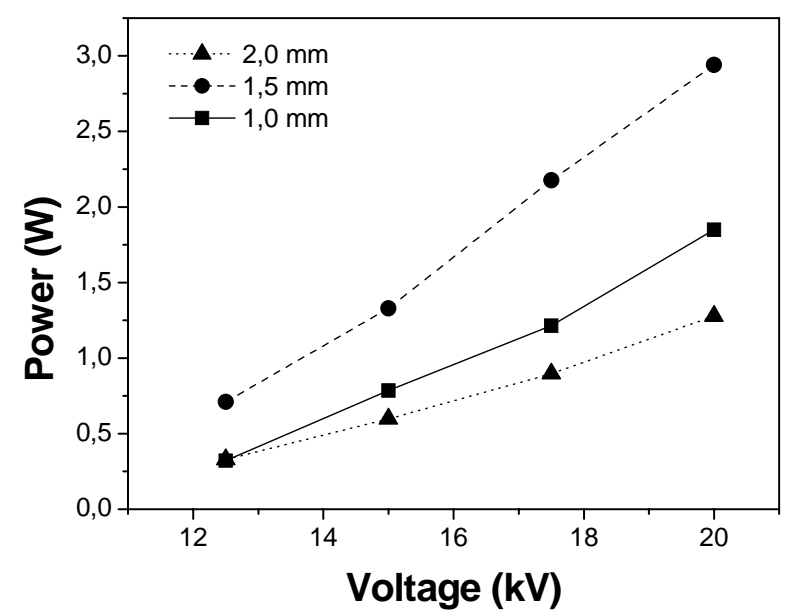

Fig. 5. Reactor power as a function of discharge voltage at different reactor gap.

$\mathrm{cm}$ is higher than the one for $1.0 \mathrm{~cm}$. This result is quite understandable taking into consideration the larger phase difference for $1.0 \mathrm{~mm}$ gap (see Fig. 4). Non-monotonic behaviour of the discharge power with the gap thickness increasing was observed for all range of operating AC voltages.

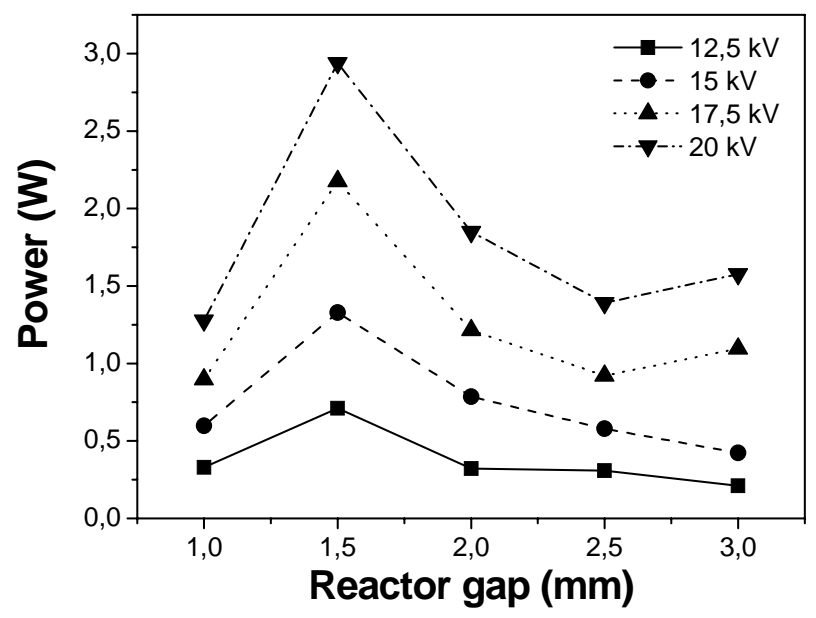

Fig. 6. Discharge power as a function of the reactor gap at different ac voltage.

Fig. 6 shows the discharge-consumed power as a function of the applied voltage for different reactor gaps. As can be seen in the whole range of applied voltages the reactor power reaches its maximal value at the same gap width of about 1.5 $\mathrm{cm}$. This value is in a reasonable agreement with the prediction from the electric circuit model (5). Assuming the cross section of the filamentary discharges much smaller than the electrodes area $\left(A_{E F}>>A\right)$, typical discharge resistivity on the order of hundreds of $\mathrm{k} \Omega . \mathrm{m}$ the second term in (5) can be neglected which leads to $x_{M A X} \approx 2 d / \kappa$. Considering our experimental parameters: gap width $d=5.0 \mathrm{~mm}$ and a commercial glass dielectric constant of about 7.6 it gives an optimal gap thickness of about $1.3 \mathrm{~mm}$ which is in accordance with the power maximum observed in Fig. 6. 
The influence of the reactor geometry on the dischargeconsumed power in silent discharges was investigated in [5] using a cylindrical DBD reactor. The results reported in this paper are in a good agreement with the empirical equation of Manley [12]. The authors of this study [5] also observed, but did not discuss, a similar non-monotonic relationship between the discharge power of DBD reactor and the radial gap thickness.

\section{CONCLUSIONS}

In this work, we have investigated the influence of DBD reactor geometry on the discharge power. The analysis of the DBD reactor equivalent circuit has shown that there should exist an optimal rector gap width, which would maximize the discharge power. The current-voltage and charge-voltage characteristics of the DBD discharge have been recorded for different reactor gap spacing. The reactor mean power has been evaluated by the parallelepiped area formed by the V-Q traces, which is directly proportional to the consumed energy per one cycle. For AC voltages in the range from $12.5 \mathrm{kV}$ to $20 \mathrm{kV}$ the maximum discharge power was obtained for rector gap of $1.5 \mathrm{~mm}$. The experimental results are in a close agreement with the predictions from the electric circuit model. The existence of an optimal reactor gap is important for the design of DBD reactors for material treatment.

\section{Acknowledgment}

One of the authors (K. G. Kostov) acknowledges the financial support from FAPESP (Fundação de Amparo à Pesquisa de Estado de São Paulo) under grant 2006/04585-0.
[1] U. Kogelschatz, IEEE Trans. Plasma Sci., 30 (2002) 1400.

[2] K. Takaki, K. Urashima, and J-S. Chang, IEEE Trans. Plasma Sci.. 32 (2004) 2175.

[3] O. Goossens, E. Dekempeneer, D. Vangeneugden, R. Van de Leest and C. Leys, Surf. Coat. Technol. 142-144 (2001) 474

[4] G. Borcia, C. A. Anderson and N. M. D. Brown, Appl. Surf. Sci. 225 (2004) 186.

[5] M. Abdel-Salam, A. Hashem, A. Yehia, A. Mizuni, A. Turky and A. Gabr, J. Phys. D: Appl. Phys., 36 (2003) 252

[6] A. Yehia and A. Mizuno, J. Appl. Phys., 98 (2005) 3051

[7] K. Takaki, Y. Hatanaka, K. Arima, S. Mikaigawa, and T. Fugi- wara, Vacuum, 83 (2009), 128

[8] R. V. Barrientos, J. P. Sotelo, M. P. Pacheco, J. S. B. Read and R. L. Callejas, Plasma Sources Sci. Technol. 15 (2006) 237

[9] K. P. Francke, R. Rudolph, and H. Miessner, Plasma Chem. Plasma Process, 23 (2003) 47

[10] G. Nersisyan and W. G. Graham, Plasma Source Sci. Tecnol., 13 (2004) 582

[11] Z. Falkenstein and J. J. Coogan, J. Phys. D, 30 (1997) 817

[12] T. C. Manley, Trans. Electrochem. Soc., 84 (1943) 83 